Min REUCHAMPS (dir.), Minority Nations in Multinational Federations. A Comparative Study of Quebec and Wallonia

\title{
Giovanni Carpinelli
}

\section{(2) OpenEdition}

1 Journals

Édition électronique

URL : http://journals.openedition.org/ress/3548

DOI : $10.4000 /$ ress.3548

ISBN : 1663-4446

ISSN : $1663-4446$

Éditeur

Librairie Droz

Édition imprimée

Date de publication : 30 novembre 2016

Pagination : 301-304

ISSN : 0048-8046

Référence électronique

Giovanni Carpinelli, « Min REUCHAMPS (dir.), Minority Nations in Multinational Federations. A

Comparative Study of Quebec and Wallonia », Revue européenne des sciences sociales [En ligne], 54-2 I

2016, mis en ligne le 22 juillet 2016, consulté le 24 septembre 2020. URL : http://

journals.openedition.org/ress/3548; DOI : https://doi.org/10.4000/ress.3548

Ce document a été généré automatiquement le 24 septembre 2020.

(c) Librairie Droz 


\title{
Min REUCHAMPS (dir.), Minority Nations in Multinational Federations. A Comparative Study of Quebec and Wallonia
}

\author{
Giovanni Carpinelli
}

\section{RÉFÉRENCE}

Min REUCHAMPS (dir.), 2015, Minority Nations in Multinational Federations. A Comparative Study of Quebec and Wallonia, London - New York, Routledge, 198 p.

1 Délicate mais nécessaire entreprise que celle d'aborder la question des fédérations multinationales d'un point de vue comparatif. C'est leur capacité à surmonter les crises dont elles sont périodiquement affectées qui attire en général l'attention des chercheurs. Les cas du Pays basque en Espagne, de la Bavière en Allemagne, du Québec au Canada, de la Flandre en Belgique et, plus récemment, de l'Écosse au Royaume-Uni sont là pour le prouver. Dans le passé, c'était presque une règle de rapprocher le Québec de la Flandre, du fait des similitudes entre les deux régions: elles représentaient les exclus, ou les faibles, au sein de leurs États respectifs. Les choses ont cependant changé depuis, la Flandre étant, en Belgique, la région de loin la plus riche et la plus peuplée. Désormais, c'est la comparaison entre le Québec et la Wallonie qui s'impose. Les auteurs de ce collectif s'y attachent en privilégiant l'analyse, menée de l'intérieur, des deux régions conçues comme étant des réalités autonomes, par rapport à celle des relations que ces deux unités entretiennent avec les structures fédérales dont elles font partie. En tant que le Québec et la Wallonie font figure de petites patries, un regard attentif doit être porté sur ce qui se passe à l'échelle locale. Telle est la prémisse des travaux réunis dans ce volume. On la retrouve aussi bien dans 
l'introduction de Min Reuchamps qu'au fil des pages et dans la conclusion de Michael Burgess.

Les événements examinés dans l'ouvrage remontent aux années 1960, quand le Québec entame sa "révolution tranquille» et la Wallonie prend conscience de son statut minoritaire du point de vue social et économique. Là commence un processus qui atteint son faîte en 1995. Au Québec, ce fut l'année du deuxième referendum sur l'indépendance : le «non» remporta une victoire arrachée de justesse. Dès lors, la tendance à l'accroissement des pouvoirs attribués au Québec dans le cadre de la Fédération s'accentua et se révéla porteuse de nombreux résultats. 1995 est également une date cruciale pour la Wallonie, puisque, pour la première fois, est élu directement un Parlement wallon. Les pouvoirs dévolus à la région s'étant encore étoffés depuis, l'ensemble de l'édifice tend à se rapprocher de celui d'un État sans s'y identifier tout à fait. Les différences entre les deux cas envisagés demeurent cependant importantes ainsi que le rappellent, respectivement, Luc Turgeon et Jean-François Caron dans les deux chapitres qui forment la première partie de l'ouvrage (Setting the stage). Le Québec représente $25 \%$ seulement de la population canadienne et, dans le pays, les francophones proprement dits sont encore moins nombreux par rapport à la population totale, puisqu'ils n'excèdent pas les $20 \%$. Dans le contexte belge, la population de la Wallonie représente $32 \%$ de la population totale, tandis que la part de francophones, avec Bruxelles, atteint $41 \%$. Fait remarquable, tout en étant un pays majoritairement flamand, la Belgique a une capitale dont $80 \%$ de la population s'exprime en français. De surcroît, la Wallonie était en fait englobée depuis longtemps dans une Belgique francophone officiellement majoritaire, ce qui contraste nettement avec la situation traditionnellement minoritaire du Québec. Qu'on ajoute à cela le vieux statut provincial du Québec et la constitution toute récente de la Wallonie en région autonome et l'on aura une idée de l'écart qui sépare les deux réalités.

3 Venons-en aux deux autres parties plus détaillées du livre. Tandis que la deuxième (trois chapitres) est consacrée à la politique (Politics), la troisième, et dernière (trois chapitres), l'est, quant à elle, aux stratégies d'action (Policies). La deuxième partie s'ouvre par une étude sémiologique (à vrai dire, les auteurs - Heidi Mercenier, Julien Perrez et Min Reuchamps - parlent de lexicométrie, de linguistique cognitive et de science politique). Il s'agit de voir quelle image de la région est offerte par les programmes électoraux des partis politiques entre 1994 et 2014. Il apparaît que la question identitaire domine au Québec, tandis qu'en Wallonie elle va de pair avec la question sociale. Les métaphores employées privilégient l'effort de construction d'une nouvelle nation au Québec, là où c'est le cadre de vie et le malaise qui sont au premier plan en Wallonie. Dans les deux contributions suivantes les partis s'effacent au profit des individus. Jérémy Dodeigne se demande si une classe politique locale est en train de se former. La Wallonie semblerait présenter de ce point de vue une plus grande mobilité ascendante. Le phénomène ne concerne toutefois qu'un petit nombre de personnes. La frontière entre l'échelon local et l'échelon supérieur garde tout son poids et les changements de statut entre l'un et l'autre sont, somme toute, rares. Sandra Breux et Vincent Jacquet, quant à eux, s'intéressent aux maires, « mayors » au Québec, «bourgmestres" en Wallonie. Leur rôle s'est vu récemment renforcé dans les deux régions. Un souci d'indépendance par rapport aux divisions partisanes et aux gouvernements centraux a fini par s'affirmer dans les deux cas. Cependant la profession du maire est plus politisée en Wallonie qu'au Québec. 
4 En ce qui concerne les stratégies d'action (troisième partie de l'ouvrage), Maxime Petit Jean s'intéresse à l'administration publique, visée par des réformes marquantes : la loi sur l'administration publique au Québec en 2000 et la naissance d'une administration centrale wallonne, dénommée Service public de Wallonie, en 2008. Les buts poursuivis étaient les mêmes: efficacité et ouverture (responsiveness) aux citoyens. La volonté d'autonomie est pourtant beaucoup plus nette au Québec. L'administration wallonne n'a été longtemps qu'une copie de l'administration belge. Elle hésite dernièrement entre le choix d'une forme locale plus autonome et l'alliance avec Bruxelles dans le cadre de la Communauté française. C'est à Philippe Hambye que revient la tâche d'aborder un thème délicat : la politique des langues. Les différences entre le Québec et la Wallonie s'accentuent à ce sujet. Dans le cas du Québec, la concurrence de l'anglais se fait sentir, tandis qu'en Wallonie le néerlandais n'a jamais exercé d'attrait véritable. Les secteurs explorés sont au nombre de quatre: communication publique, éducation, politiques d'immigration et planification linguistique. Seul le Québec a développé une politique des langues cohérente et transversale, notamment avec la Charte de la langue française qui vise à assurer la priorité du français dans tous les domaines publics d'expression verbale. On peut ajouter qu'une telle politique a porté ses fruits. Enfin Stéphane Paquin, Marine Kravagna et Min Reuchamps consacrent un dernier chapitre aux relations internationales menées par chacune des deux régions. L'exercice d'une diplomatie parallèle a conduit le Québec à promouvoir une politique étrangère distincte de celle du Canada. En revanche, en Belgique on a assisté le plus souvent à une influence conjointe des deux acteurs francophones, Wallonie et Communauté française, sur les choix de l'État fédéral.

5 Un bilan de l'entreprise réalisée par Minority Nations tend à montrer que le choix de s'en tenir principalement au niveau régional dans l'analyse n'a pas été toujours heureux. Il est vrai que le Québec et la Wallonie sont des nations en marche, mais contre quels adversaires? Qu'est-ce qui a empêché le Québec et la Wallonie d'aller plus loin sur le chemin d'une indépendance totale ? Et, d'abord, est-il si évident que la Wallonie aspire à l'indépendance? Dans cet ouvrage même, Jean-François Caron décrit comme suit l'état actuel de l'opinion locale : « il n'est pas surprenant aujourd'hui de trouver qu'une vaste majorité de Wallons s'identifient davantage à la Belgique qu’à leurs autres entités culturelles (que ce soit la région wallonne ou la Communauté française) » (p. 32). Quant au Québec, l'option séparatiste à été sans conteste rejetée lors du referendum de 1995 par un nombre considérable de francophones (40\% environ) et par des minorités qui se sentaient menacées par une telle perspective (voir le cas des féministes anglophones cité par Luc Turgeon, p.19). L'on dirait que des figurants inattendus sont venus perturber le scénario d'une pièce peut-être trop simpliste : les Canadiens, qui existent, et les Belges, qui existent aussi. Ce sont eux qui s'opposent à un fractionnement plus poussé de leurs États. Que ce soit au nom de la neutralité scientifique ou pour d'autres raisons non explicitées, les dégâts provoqués par un morcellement excessif du pouvoir ne se voient pas accorder une place de choix dans cet ouvrage. Or, les attentats terroristes de Bruxelles du 22 mars 2016 sont là pour nous rappeler que le souci de ménager les susceptibilités locales peut devenir un obstacle majeur dans la prévention des atteintes à la sécurité collective. 


\section{AUTEURS}

\section{GIOVANNI CARPINELLI}

Turin, Fondazione Istituto Piemontese Antonio Gramsci 